\title{
Emitting Behaviors of 1-Acetylaminopyrene Dispersed in Poly(vinyl acetate) Film
}

\author{
Hiroki Takahashi and Hideyuki Nakano* \\ Department of Applied Chemistry, Muroran Institute of Technology, \\ 27-1 Mizumoto-cho, Muroran, Hokkaido 050-8585, Japan \\ *nakano@mmm.muroran-it.ac.jp
}

\begin{abstract}
In order to create a new stimuli-responsible emitting system, we have investigated the thermal phase behaviors and emitting properties of the film of 1-acetylaminopyrene (AAPy) dispersed in poly(vinyl acetate) (PVAc). By heating the amorphous film obtained by spincoating method, crystallization took place at ca. $130^{\circ} \mathrm{C}$ followed by melting and/or resolution to recover the homogeneous film over $200{ }^{\circ} \mathrm{C}$. Both crystalline and amorphous phases could be reversibly obtained at room temperature by annealing the film at $130^{\circ} \mathrm{C}$ and $245^{\circ} \mathrm{C}$, respectively. Such phase transition to control the state of the film at room temperature allowed the reversible fluorescence color change of the film due to the difference of emission spectra between amorphous and crystalline phases.

Keywords: Stimuli-responsible system, Fluorescence, Phase transition, 1-Acetylaminopyrene, Poly(vinyl acetate)
\end{abstract}

\section{Introduction}

Stimuli-responsible emitting materials and molecular systems are the candidates for practical applications such as visible sensors and fluorescence switches triggered by external stimuli. Creation of such systems and investigation of their properties responsible to external stimuli to clarify the mechanism are attractive subjects. Recent example of interest is mechanochromic fluorescent materials that exhibit reversible emission color changes of the solid emitting materials by mechanical stimuli such as grinding [1-6]. A variety of mechanochromic fluorescent materials have been reported and the phenomena were generally believed to be due to change in intermolecular interactions caused by altering their molecular arrangements in the crystalline state induced by mechanical stress. We have been reported two series of mechanochromic fluorescent materials, i.e., amorphous molecular materials based on diarylaminobenzaldehyde [7-10] and 1alkanoylaminopyrenes [11-12]. With regard to the latter system, fluorescent color of recrystallized sample of 1-alkanoylaminopyrens, including 1acetylaminopyrene (AAPy), changed bluish purple to yellowish green by grinding due to formation of crystal defects which allowed to form excimer [11]. In addition, we have found that 1-alkanoylaminopyrenes adsorbed onto cellulose papers also exhibited mechanochromic fluorescence that are somewhat different from those observed in their crystalline systems [12]. Thus, emitting properties of 1-alknoylaminopyrenes were expected to be controlled by altering the surrounding environments.

By the way, we have been reported that the binary films composed of quaternary ammonium and phosphonium salts together with poly(vinyl acetate) (PVAc) exhibited thermally reversible phase separation-dissolution behaviors and the states at room temperature were controllable by thermal histories [13]. In addition, reversible display switching and fluorescence modulation were demonstrated by controlling the states of the films by thermal treatments [13]. Thus, PVAc is expected to act as a matrix for switching the environment by thermal treatments.

In order to create a new stimuli-responsible emitting system, we have investigated the thermal behavior and emitting properties of the film of AAPy dispersed in PVAc in the present study and have found that the emitting behavior was switchable by appropriate thermal treatments. 


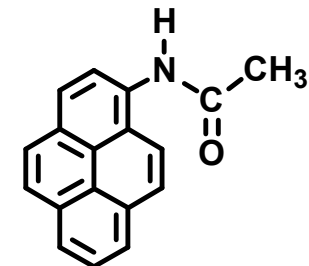

AAPy

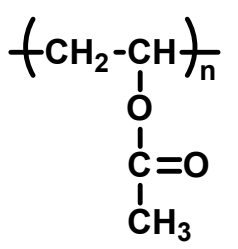

PVAc

\section{Experimental}

Sample films were prepared by spin-coating onto transparent glass substrates from THF solutions of AAPy and PVAc with a ratio of 5:95 (w/w). Polarizing microscopy was performed in a crossed nicol state with an ECLIPSE Ni (Nikon) microscope fitted with a TH-600PM hot stage (Linkam). Differential scanning calorimetry (DSC) was carried out by means of a Seiko DSC6220. X-Ray diffraction measurements for the films were performed using a Rigaku FR-E X-ray diffractometer with an R-AXIS IV 2D detector (Rigaku, Japan) in the same manner as the grazingincidence X-ray scattering measurement [14]. Fluorescent spectra were measured with an FP-8300 spectrofluorometer (JASCO Co.) at room temperature.

\section{Results and discussion}

A smooth and transparent film was obtained by spin-coating method from an THF solution of AAPy and PVAc. The resulting spin-coated film was confirmed to be in amorphous state by polarizing microscopy. When the film was heated up to $130{ }^{\circ} \mathrm{C}$, crystallization was found to take place. When the sample was cooled to room temperature, crystalline phase was maintained confirmed by polarizing microscopy in a crossed nicol state as shown in Fig. 1a. When the film was heated up to $245^{\circ} \mathrm{C}$, melting of the crystal and/or resolution took place to give homogeneous film. By cooling the film on standing, the homogeneous amorphous state was again obtained at room temperature, the polarizing microscope image being in dark (Fig. 1b). Thus, the state of the film could be switching between amorphous and crystalline ones by the history of heat treatments.

Such phase transition was confirmed by differential scanning calorimetry (DSC) and X-ray diffraction (XRD). Figure 2 shows a DSC curve for the second heating of the mixture of AAPy and PVAc. When the sample was heated, an obvious glass transition phenomenon was observed at ca. $40{ }^{\circ} \mathrm{C}$, being almost identical to the glass transition temperature of PVAc. On further heating, an a)

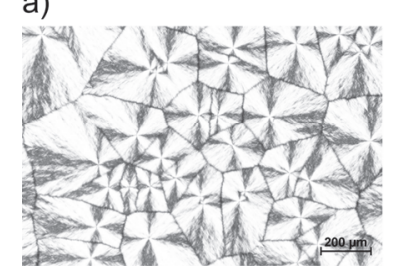

b)

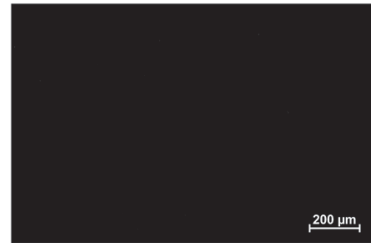

Fig. 1. Polarizing microscope images of AAPy-PVAc film after annealing at a) $130{ }^{\circ} \mathrm{C}$ and b) $245{ }^{\circ} \mathrm{C}$ in a crossed nicol state.

exothermic peak was observed at ca. $130{ }^{\circ} \mathrm{C}$, attributable to crystallization. Then, a broad endothermic peak was observed up to ca. $200{ }^{\circ} \mathrm{C}$ maybe due to melting and/or resolution. As shown in inset of Fig. 2, the sample film after annealing at $130{ }^{\circ} \mathrm{C}$ exhibited XRD peaks due to crystalline phase and no XRD peak was observed for the film after annealing at $245{ }^{\circ} \mathrm{C}$ suggesting the film to be in homogeneous amorphous state. It is noted that the XRD patterns observed after annealing at $130^{\circ} \mathrm{C}$ was almost identical to that for AAPy crystal [11], therefore, AAPy crystals were produced by annealing the amorphous film at $130{ }^{\circ} \mathrm{C}$.

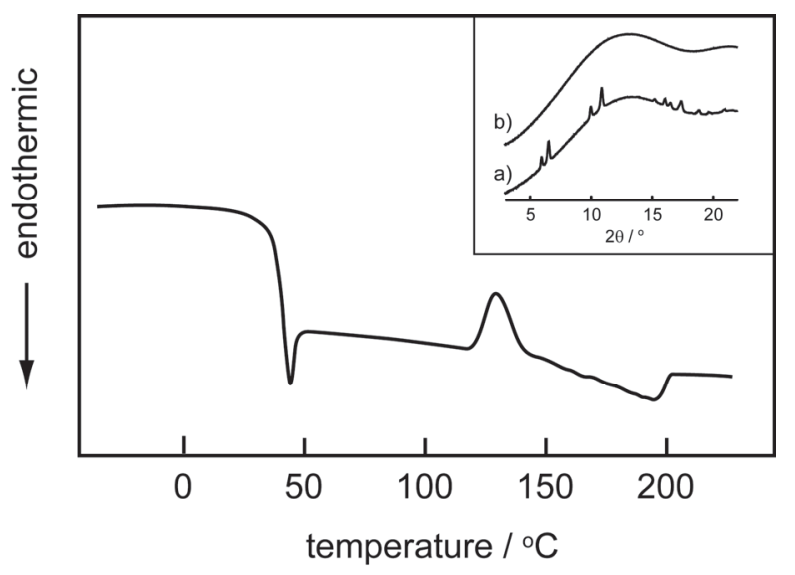

Fig. 2. DSC curve of the mixture of AAPy and PVAc $(5: 95 \mathrm{w} / \mathrm{w})$. Inset: XRD patterns of AAPy-PVAc film after annealing at a) $130{ }^{\circ} \mathrm{C}$ and b) $245^{\circ} \mathrm{C}$.

In the next stage, emission spectral changes of the AAPy-PVAc film by heat treatments were investigated. With regard to the as-prepared film by spin-coating, monomeric emission band around $400 \mathrm{~nm}$ together with excimer emission band around $470 \mathrm{~nm}$ was observed (Fig. 3a). When the film was annealed at $130{ }^{\circ} \mathrm{C}$, the excimer emission was found to decrease for fluorescence spectrum (Fig. 3b), resulting in emission color change from light blue to bluish purple. When the sample was annealed at $245^{\circ} \mathrm{C}$, the emission band for excimer 
again increased (Fig. 3c) and the emission color at room temperature recovered to light blue. Such emission color changes were found to take place repeatedly by alternate annealing at $130{ }^{\circ} \mathrm{C}$ and $245{ }^{\circ} \mathrm{C}$. Relative emission intensity at $470 \mathrm{~nm}$ attributable to excimer emission decreased by annealing at $130{ }^{\circ} \mathrm{C}$, followed by increasing by annealing at $245^{\circ} \mathrm{C}$ as shown in the inset of Fig. 3.

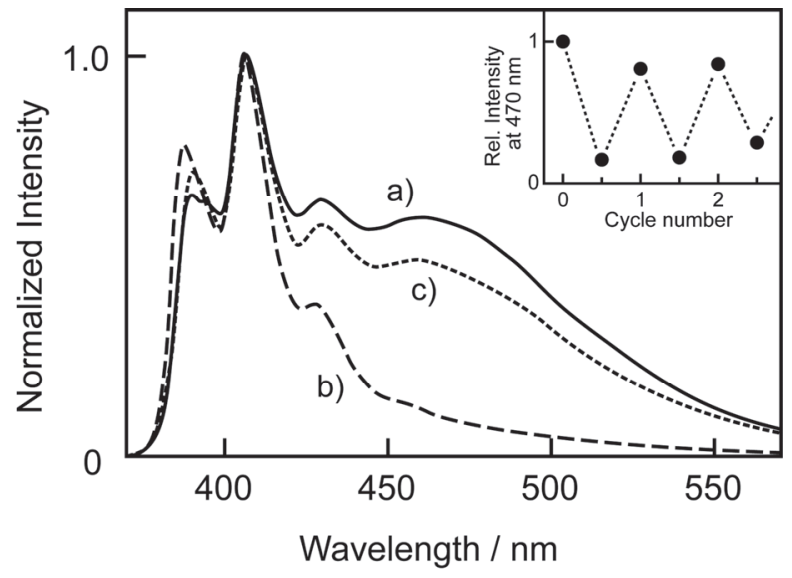

Fig. 3. Emission spectral changes of AAPy-PVAc film at room temperature. a) as-prepared film. b) after annealing at $130{ }^{\circ} \mathrm{C}$. c) after annealing at $245{ }^{\circ} \mathrm{C}$. Inset: Chang in relative emission intensity at $470 \mathrm{~nm}$ by alternate annealing cycle at $130^{\circ} \mathrm{C}$ and $245^{\circ} \mathrm{C} . \quad \lambda_{\text {ex }}: 350$ nm.

We have reported that fluorescence spectrum of AAPy crystal was shifted to longer-wavelength region relative to that in solution and concluded that the AAPy molecules stacked in a J-aggregate manner in the crystalline state resulting in red-shifts of the electronic absorption and emission spectra [11]. As described above, AAPy crystals were produced by annealing of the AAPy-PVAc film at $130{ }^{\circ} \mathrm{C}$; however, the emission spectrum of the annealed AAPy-PVAc film (Fig. 3b) was identical to the spectrum of AAPy in solution, being different from that of AAPy crystal [11]. It was assumed that considerable amount of AAPy molecules remain to dissolve in PVAc without crystallization after annealing at $130{ }^{\circ} \mathrm{C}$ and then the fluorescence from such AAPy molecules in PVAc became dominant, resulting in fluorescence spectrum similar to that in solution. Further studies regarding the phase-behaviors and emission properties were in progress.

\section{Conclusion}

In order to create a new stimuli-responsible emitting system, we have prepared the AAPy dispersed PVAc film and investigated its thermal phase behaviors and emitting properties. It was found that the film took both crystalline and amorphous states at room temperature according to the history of heat treatments, that is, the film took crystalline and amorphous states after annealing at $130{ }^{\circ} \mathrm{C}$ and $245^{\circ} \mathrm{C}$, respectively. By using such phase behaviors, reversible emission color change of the film was achieved. Thus, 1alkanoylaminopyrene-dispersed PVAc films were potential candidates for emission color switching system.

\section{Acknowledgement}

This work was partly supported by JSPS KAKENHI Grant Number JP26107006 in Scientific Research on Innovative Areas "Photosynergetics" and by Nanotechnology Platform Program (Molecule and Material Synthesis) of the Ministry of Education, Culture, Sports, Science and Technology (MEXT), Japan. We thank Prof. S. Nagano (Nagoya University Venture Business Laboratory) for performing XRD and his helpful discussion.

\section{References}

1. Y. Sagara and T. Kato, Nature Chem., 1 (2009) 605.

2. Y. Ooyama, Y. Kagawa, H. Fukuoka, G. Ito, and Y. Harima, Eur. J. Org. Chem., 2009, 5321.

3. G. Zhang, J. Lu, M. Sabat, and C. L. Fraser, J. Am. Chem. Soc., 132 (2010) 2160.

4. Z. Chi, X. Zhang, B. Xu, X. Zhou, C. Ma, Y. Zhang, S. Liu, and J. Xu, Chem. Soc. Rev., 41 (2012) 3878 .

5. Y. Sagara, T. Komatsu, T. Ueno, K. Hanaoka, T. Kato, and T. Nagano, J. Am. Chem. Soc., 136 (2014) 4273.

6. M. Zheng, D. T. Zhang, M. X. Sun, Y. P. Li, T. L. Liu, S. F. Xue, W. J. Yang, J. Mater. Chem. C, 2 (2014) 1913.

7. K. Mizuguchi, H. Kageyama, and H. Nakano, Mater. Lett., 65 (2011) 2658.

8. K. Mizuguchi and H. Nakano, Dyes Pigm., 96 (2013) 76 .

9. K. Okoshi and H. Nakano, J. Photopolym. Sci. Tech., 27 (2014) 535.

10. S. Manabe, E. Nagata, and H. Nakano, Rapid Commun. Photosci., 3 (2014) 38.

11. E. Nagata, S. Takeuchi, T. Nakanishi, Y. Hasegawa, Y. Mawatari, and H. Nakano, ChemPhysChem, 16 (2015) 3038.

12. E. Nagata, T. Ara, and H. Nakano, Dyes Pigm., 
141 (2017) 48.

13. R. Ichikawa, D. Watanabe, Z. Hoshi, and H. Nakano, Kobunshi Ronbunshu, 71 (2014) 475.
14. M. Sano, S. Nakamura, M. Hara, S. Nagano, Y. Shinohara, Y. Amemiya, and T. Seki, Macromolecules, 47 (2014) 7178. 